\title{
Distinct Functional Activity of the Precuneus and Posterior Cingulate Cortex During Encoding in the Preclinical Stage of Alzheimer's Disease
}

\author{
Lorena Rami ${ }^{\mathrm{a}, \mathrm{c}}$, Roser Sala-Llonch ${ }^{\mathrm{b}}$, Cristina Solé-Padullés ${ }^{\mathrm{a}}$, Juan Fortea ${ }^{\mathrm{a}}$, Jaume Olives ${ }^{\mathrm{a}}$, \\ Albert Lladón $^{\mathrm{a}, \mathrm{c}}$, Cleofe Peña-Gómez ${ }^{\mathrm{b}}$, Mircea Balasa ${ }^{\mathrm{a}}$, Bea Bosch ${ }^{\mathrm{a}, \mathrm{c}}$, Anna Antonell ${ }^{\mathrm{a}, \mathrm{c}}$, \\ Raquel Sanchez-Valle ${ }^{\mathrm{a}, \mathrm{c}}$, David Bartrés-Faz ${ }^{\mathrm{b}}$ and Jose L. Molinuevo ${ }^{\mathrm{a}, \mathrm{c}, *}$ \\ ${ }^{a}$ Alzheimer's Disease and Other Cognitive Disorders Unit, Neurology Service, Hospital Clínic, Barcelona, Spain \\ ${ }^{\mathrm{b}}$ Department de Psiquiatria i Psicobiologia Clinica, Universitat de Barcelona, Barcelona, Spain \\ ${ }^{\mathrm{c}}$ Institut d'Investigacions Biomèdiques August Pi i Sunyer (IDIBAPS), Barcelona, Spain
}

Accepted 16 April 2012

\begin{abstract}
In this study functional magnetic resonance imaging (fMRI) is used to investigate the functional brain activation pattern in the preclinical stage of $\mathrm{AD}$ (pre-AD) subjects during a visual encoding memory task. Thirty subjects, eleven in the pre-AD stage, with decreased cerebrospinal fluid levels of $A \beta_{42}(<500 \mathrm{pg} / \mathrm{ml})$, and 19 controls with normal $A \beta_{42}$ levels (CTR) were included. fMRI was acquired during a visual encoding task. Data were analyzed through an Independent Component Analysis (ICA) and region-of-interest-based univariate analysis of task-related BOLD signal change. From the ICA decomposition, we identified the main task-related component, which included the activation of visual associative areas and prefrontal executive regions, and the deactivation of the default-mode network. The activation was positively correlated with task performance in the CTR group $(p<0.0054)$. Within this pattern, subjects in the pre-AD stage had significantly greater activation of the precuneus and posterior cingulate cortex during encoding. Subjects in the pre-AD stage present distinct functional neural activity before the appearance of clinical symptomatology. These findings may represent that subtle changes in functional brain activity precede clinical and cognitive symptoms in the AD continuum. Present findings provide evidence suggesting that fMRI may be a suitable biomarker of preclinical AD.
\end{abstract}

Keywords: Alzheimer's disease, biomarkers, cerebrospinal fluid, cognition, diagnosis, functional magnetic resonance imaging, preclinical phases

Supplementary data available online: http://dx.doi.org/10.3233/JAD-2012-120223

\footnotetext{
*Correspondence to: José Luis Molinuevo, M.D., Alzheimer's disease and Other Cognitive Disorders Unit, Neurology Service, Hospital Clínic, Villarroel 170. 08036, Barcelona, Spain. Tel.: +34 932275785; Fax: +34 932275783; E-mail: jlmoli@ clinic.ub.es.
}

\section{INTRODUCTION}

The main pathological lesions in Alzheimer's disease (AD) are neurofibrillary tangles and senile plaques formed by neuronal accumulations of abnormal tau filaments and extracellular deposits of amyloid fibrils. Cerebrospinal fluid (CSF) studies measuring 
amyloid- $\beta_{42}\left(A \beta_{42}\right)$ and tau protein levels in $\mathrm{AD}$ patients with confirmed pathology have demonstrated that abnormal levels of both biomarkers constitute a specific signature of the underlying AD-related pathology [1].

The neuropathological burden of AD occurs very early during the course of the disease, preceding the appearance of clinical symptoms [2]. It has been shown that AD pathology begins a decade before the appearance of symptoms, or in some cases even earlier [2,3]. Recent studies have demonstrated that approximately $25 \%$ of older non-demented individuals have $A \beta_{42}$ deposition, corroborating the data observed in studies with postmortem confirmation $[4,5]$. The significance and prognostic value of AD pathological lesions in cognitively healthy individuals is still under debate. Some authors hold that these lesions are irrelevant, while others regard them as part of the pathophysiology of the disease, indicating that these individuals are at the preclinical stage $[6,7]$.

Recently, it has been postulated that the preclinical stage of the disease is a long asymptomatic period during which the pathophysiological process progresses. Subjects in the preclinical stage of AD (pre-AD) have been defined as individuals who have evidence of early AD pathological changes but who do not meet clinical criteria for mild cognitive impairment (MCI) or dementia [8]. Furthermore, individuals with biomarker evidence of $\mathrm{AD}$ are supposed to have an increased risk for developing cognitive and behavioral impairment [8]. Two groups have been distinguished within pre$\mathrm{AD}$; presymptomatic AD subjects and asymptomatic at-risk state for AD [9]. Presymptomatic subjects apply to individuals belonging to rare monogenic AD families who will develop AD if they live up until the age of disease onset in their family; by contrast asymptomatic at risk do not have a genetically determined risk and can be identified in vivo by evidence of amyloidosis of the brain (either through PET or CSF). Furthermore, cognitive performance in this latter group is related with CSF $A \beta_{42}$ values [10], suggesting that the preclinical stage may be different from normal aging. Therefore, determining brain functional changes in pre-AD subjects may be useful to support the hypothesis that the preclinical state of AD is distinct from normal aging. In addition, characterizing the preclinical state from the perspective of imaging and biomarkers may help to design future trials with disease-modifying therapies that target this stage of the disease.

Functional MRI (fMRI) is well suited in order to study patterns of brain activity. Interestingly, and particularly based on connectivity analyses, fMRI is suitable to capture the hypercomplex organization of the human brain and their subtle dysfunctions before cognitive alterations become apparent, hence resulting in a dynamic and sensitive marker for $\mathrm{AD}$ [11]. Converging evidence suggest that cerebral amyloidosis in pre-AD phases is associated with structural and functional changes in the medial parietal areas [12], considered core regions of the default mode network (DMN), suggesting that these are key regions of early brain dysfunction associated to AD process. Sperling et al. [13] demonstrated that high levels of amyloid deposition were associated with aberrant DMN activity, similar to the pattern of dysfunction reported in AD patients. Although previous studies have described changes in brain function in healthy subjects with positive PIB [12-15], there are no fMRI in healthy subjects with pathological CSF $A \beta_{42}$ values.

\section{Objectives and hypothesis}

The aim of this study was to use fMRI to determine whether the functional brain activation pattern during encoding is different among pre-AD subjects, classified according to the presence of pathological $\mathrm{A} \beta_{42}$ CSF levels. We hypothesize that pre-AD subjects will present a distinct pattern of activation from healthy controls during encoding, thus suggesting that this preclinical state is functionally different from healthy aging. To our knowledge most of the literature conducted in subjects with pre-AD characteristics incorporating fMRI technology has been based in individuals with evidence of amyloid deposition on PET imaging and in resting state fMRI investigations $[12,14,16]$ with fewer data obtained during cognitive challenge paradigms [13]. In the present study and according to the findings derived in these previous reports we expected that functional abnormalities mainly in posterior cingulate cortex/precuneus regions would be apparent during memory encoding in our pre-AD defined by abnormal concentrations of CSF biomarkers and that functional alterations would correlate with subsequent memory performance.

\section{MATERIAL AND METHODS}

\section{Study population}

Thirty subjects were recruited from the Alzheimer's disease and other cognitive disorders unit at the Hospital Clinic, Barcelona, Spain. All subjects present normal cognition and met the following criteria: Mini Mental State Examination (MMSE) scores above 
24, objective cognitive performance within the normal range (performance within 1.5 SD) in all tests from a specific test battery (see below), clinical dementia rating (CDR) scale score of 0 , and no significant psychiatric symptoms or previous neurological disease. Pre-AD subjects were defined [9] as cognitively preserved subjects with decreased CSF A $\beta_{42}$ $(<500 \mathrm{pg} / \mathrm{ml}$ ) levels. 11 pre-AD and 19 controls (CTR), with normal $A \beta_{42}$ levels, were included. All subjects underwent clinical and neuropsychological assessment, magnetic resonance imaging (MRI), and lumbar puncture (LP). The study was approved by the local ethics committee and all participants gave written informed consent prior to enrolment.

\section{Determination of CSF biomarkers}

Subjects underwent LP between 9 a.m. and 12 p.m. Ten $10 \mathrm{~mL}$ of CSF was collected. The samples were centrifuged and stored in polypropylene tubes at $-80^{\circ} \mathrm{C}$ within the first hour after extraction. Levels of $A \beta_{1-42}$, total tau (t-tau), and phosphorylated tau at threonine-181 (p-tau) were measured by enzymelinked immunosorbent assay kits (Innogenetics, Ghent, Belgium). A cut off of $500 \mathrm{pg} / \mathrm{ml}$ was used to dichotomize the sample into $A \beta$-positive and $A \beta$ negative subjects [17]. These cut off scores are in agreement with the internal values of our laboratory [18].

\section{Neuropsychological and functional assessment}

Participants were administered a one-hour neuropsychological battery test by a trained neuropsychologist. The battery included memory, language, praxis, visual perception and frontal functions assessment. All neuropsychological scores were adjusted for age and educational level. Normative neuropsychological data and the neuropsychological battery have been described in detail elsewhere [19].

\section{MRI acquisition}

Subjects were scanned using a 3T Siemens Magnetom Trio Tim (Siemens, Erlangen, Germany) at the Center for Image Diagnosis of the Hospital Clinic (CDIC) in Barcelona. During the fMRI protocol, 225 $\mathrm{T} 2 *$-weighted volumes were acquired $(\mathrm{TR}=2000 \mathrm{~ms}$, $\mathrm{TE}=16 \mathrm{~ms}, 40$ slices per volume, slice thickness $=3 \mathrm{~mm}$, voxel size $=1.7 \times 1.7 \mathrm{~mm}$, distance factor $=25 \%, F O V=220 \mathrm{~mm}$, matrix size $=128 \times 128$ ). A high-resolution T1-weighted MP-RAGE image was also acquired $(\mathrm{TR}=2300 \mathrm{~ms}, \mathrm{TE}=2.98 \mathrm{~ms}, 240$ sagittal slices, FOV $=256 \mathrm{~mm}$; matrix size $=256 \times 256$; slice thickness $=1 \mathrm{~mm}$ ).

\section{Encoding fMRI session}

The cognitive paradigm consisted in a block design measuring visual encoding memory adapted from a previous study by our group [20]. The selected images were emotionally neutral and were selected according to color and form. We used an adaptation of a visual encoding task previously used by our group [20]. The task consisted of a 15-block design paradigm with alternating "fixation", "repeated", and "encoding" conditions. The whole encoding fMRI session lasted 7 minutes and 30 seconds, in which each block lasted 30 seconds and was repeated five times. The task started with a 'fixation' block, which consisted of the presentation of a white fixation cross situated in the center of a black screen. Following this block the 'repeated' condition started, which comprised a repeated presentation of the same image (10 times). The encoding phase ('encoding' block) consisted of a presentation of 10 novel pictures, with a total of 50 new photographs. All stimuli (fixation, repeated, encoding) were presented for two seconds with an interstimulus interval of one second. Before entering the scanning, subjects were told to pay attention to the pictures that would be appearing in the screen, since they would be asked about them afterwards, but without specifying the type of assessment.

\section{Memory testing after fMRI session}

Immediately after exiting the scanner, subjects were tested for their memory of the novel scenes in a twoalternative forced-choice task in which they had to decide which picture from a pair of scenes (presented on the left or right of a computer screen) they had previously seen. Once the patients pressed the response button (left or right arrow depending on the position of the photograph), another pair of images was presented, with a total of 50 pairs. The photographs were matched for content (between old and new photographs) during the recognition task. Recognition memory was thus obtained with a maximum score of 50 . The side of presentation on the screen of the encoded picture was randomized so that the scenes appeared the same number of times on each side. In addition, the order of presentation of the encoded pictures was randomized with respect to the encoding phase. All the images were presented using the Presentation software 
program (version 0.50, Neurobehavioral Systems, 2002; http://www.neurobs.com/) implemented on a laptop computer.

\section{Independent component analysis of fMRI data}

A tensor-ICA (TICA) decomposition of the fMRI datasets was performed as implemented in MELODIC [21] version 3.10, part of the FSL software (http://www.fmrib.ox.ac.uk/fsl/) in order to find common spatio-temporal patterns of signal oscillations. First, each fMRI dataset was corrected for motion using MCFLIRT [22]. Then, nonbrain voxels were removed using BET [23], spatial smoothing with a gaussian kernel of FWHM $=6.0 \mathrm{~mm}$ was applied, and the entire $4 \mathrm{D}$ dataset was normalized using the grand mean intensity. High pass temporal filtering $($ sigma $=50 \mathrm{~s}$ ) was used in order to restrict for task-related temporal patterns, and $4 \mathrm{D}$ sets were finally registered to the MNI152 template. After this preprocessing, fMRI analysis of the task was carried out using TICA. With this procedure, fMRI data is decomposed into a set of spatio-temporally independent components (IC). Spatial maps of each IC were then thresholded using a Gaussian/gammamixture model and represented on the MNI standard template.

MELODIC also performed a post-hoc analysis on the estimated temporal domain for each component. A general linear model (GLM) matrix was introduced in order to model the task time-series and to evaluate components related to each contrast ('encoding' > 'repeated' images and 'encoding' > 'fixation'). The main component associated with the encoding of new images was used to define a set of spherical regions of interest (ROIs) (of $6 \mathrm{~mm}$ radius) centered in the peaks of the main activated and deactivated brain areas during the task.

\section{ROI-based study of task-associated brain activity}

Task-fMRI data was analyzed using a model-driven approach as implemented in FMRI Analysis Tool (FEAT) from FSL. Time-series statistical analysis was carried out using a GLM approach with local autocorrelation correction [24]. Three regressors were used to model the different task blocks ('encoding', 'repeated', and 'fixation'), three additional regressors, modeling their first derivatives were introduced as nuisance variables. Individual contrast images were computed from the preprocessed functional data as follows: encoding $>$ repeated images, encoding $>$ fixation and fixation $>$ task ('encoding' + 'repeated' images).
In order to measure task-related changes in BOLD response, we obtained the $\%$ signal change within each ROIs for each of the contrasts defined above, using FEATQUERY tool.

The values obtained were compared between groups with independent samples $t$-test analysis, performed in SPSS (Statistical Package for Social Sciences, v.16, Chicago, IL, USA).

\section{RESULTS}

\section{Demographic, clinical, and CSF characteristics}

Demographic and clinical characteristics are summarized in Table 1. There were no differences in age between CTR and pre-AD subjects. Mean and SD of CSF biomarkers are shown in Table 1. As far as neuropsychological features were concerned, there were no significant differences between groups on any cognitive test or in the presence of subjective memory complaints. There were also no differences in the APOE status between groups $(p>0.05)$.

\section{Performance on functional magnetic resonance imaging activation task}

Recognition performance on the post scan memory testing ranged from $60 \%$ to $100 \%$ accuracy (mean correct, $81.9 \%$; SD, 11.1\%). There were no significant differences between CTR and pre-AD subjects $(82.4 \%$ versus $80.8 \%$ ). Performance on this test was not correlated with subjects' age, education, or degree of clinical impairment, as measured by the CDR-SB $(p>0.05)$.

\section{fMRI results}

The ICA revealed a main task-related component which was statistically significant for the novel images $>$ fixation and for the novel $>$ repeated images $(p<0.00001$ in both cases). The spatial map of this component (see Fig. 1A) included the activation of the occipital pole, the lateral occipital cortex, the lingual gyrus and fusiform gyrus (BA17, 18, 19], as well as the middle and inferior frontal gyrus and the precentral gyrus (BA6, 9, 46]. It also included task-related deactivations in areas of the default-mode network [25] such as the precuneus cortex and cingulate gyrus (BA7, 23,31 ), and the angular gyrus, supramarginal gyrus, and lateral occipital cortex (BA19, 39, 40). The activation of this component was positively correlated with task performance only in the CTR group $(r=0.531$, $p=0.016$ ). 
Table 1

Demographic, CSF and neuropsychological differences between CTR and pre-AD

\begin{tabular}{|c|c|c|c|c|c|c|}
\hline & \multicolumn{2}{|c|}{ CTR $(n=19)$} & \multicolumn{2}{|c|}{ Pre-AD $(n=11)$} & \multirow[b]{2}{*}{$t$} & \multirow[b]{2}{*}{$p$} \\
\hline & Mean & SD & Mean & SD & & \\
\hline Age (Years) & 67.0 & 5.5 & 71.8 & 9.3 & 1.8 & $\overline{\text { n.s }}$ \\
\hline Education (Years) & 10.9 & 4.5 & 8.5 & 4.5 & 1.3 & n.s \\
\hline Gender (\% women) & $68 \%$ & & $63 \%$ & & $0.8^{+}$ & n.s \\
\hline$A \beta_{1-42}$ & 670.1 & 131.5 & 372.9 & 93.7 & 6.8 & $* *$ \\
\hline t-tau & 356.8 & 224.8 & 452.5 & 566.5 & 0.6 & n.s \\
\hline p-tau & 63.7 & 30.7 & 71.7 & 57.3 & 0.5 & n.s \\
\hline M@T & 46.2 & 3.2 & 44.0 & 5.7 & 1.2 & n.s \\
\hline MMSE & 28.3 & 1.2 & 28.1 & 1.6 & 0.2 & n.s \\
\hline CERAD_learning & 19.0 & 3.3 & 17.7 & 2.9 & 1.2 & n.s \\
\hline CERAD_recall & 5.7 & 1.5 & 5.2 & 1.9 & 1.2 & n.s \\
\hline CERAD_Recognition & 19.2 & 0.8 & 18.3 & 1.6 & 2.2 & n.s \\
\hline FCSRT- learning & 25.8 & 6.1 & 21.4 & 7.9 & 1.0 & n.s \\
\hline FCSRT-total learning & 41.3 & 7.1 & 39.5 & 7.5 & 0.4 & n.s \\
\hline FCSRT-recall & 10.1 & 1.6 & 8.7 & 3.5 & 0.3 & n.s \\
\hline FCSRT-total recall & 14.4 & 1.4 & 13.4 & 2.8 & 0.4 & n.s \\
\hline Visual memory (CERAD) & 8.9 & 2.1 & 8.1 & 2.5 & 1.6 & n.s \\
\hline Boston Naming Test & 52.0 & 4.0 & 51.7 & 5.1 & 0.8 & n.s \\
\hline Semantic Fluency & 18.7 & 4.6 & 17.8 & 5.0 & 0.1 & n.s \\
\hline PDT & 14.0 & 1.0 & 14.5 & 0.5 & 1.4 & n.s \\
\hline VOSP-letters & 19.3 & 0.9 & 19.8 & 0.3 & 1.1 & n.s \\
\hline CERAD_praxis & 10.1 & 1.4 & 10.3 & 1.1 & 0.2 & n.s \\
\hline TMT-A & 52.3 & 18.5 & 56.2 & 19.1 & 0.1 & n.s \\
\hline TMT-B & 161.3 & 80.3 & 158.4 & 85.9 & 0.3 & n.s \\
\hline Verbal Fluency (FAS) & 32.6 & 14.3 & 26.3 & 13.2 & 0.9 & n.s \\
\hline Digit symbol & 40.8 & 20.0 & 50.2 & 12.6 & 1.1 & n.s \\
\hline
\end{tabular}

${ }^{* *} p<0.005 ;{ }^{+} \mathrm{X}^{2}$

CTR: controls; AD: Alzheimer's disease; MT: Memory alteration test; FCSRT: Free and cued selective reminding test; PDT: perceptual digital test; TMT-A: trail making test A; TMT-B: trail making test B.

A total number of 9 spherical ROIs were defined from the spatial map of the IC described above. Six of them were associated with task-related activations, and three with task-related deactivations (Fig. 1B and Table 2). The percent signal change between the encoding for novel images and the repeated images in the defined ROIs was calculated in both controls and pre-AD (Supplementary Table; available online: http://www.j-alz.com/issues/31/vol313.html\#supplementarydata01). We only observed a significant BOLD response in the pre-AD group compared to CTR within the precuneus and posterior cingulate cortex ROI ( $t=3.12, p=0.004$, Fig. 1C). There was a tendency for negative correlation between the activity in the precuneus and posterior cingulate cortex during encoding and the task performance $(r=-0.315, p=0.085)$. The $\%$ of signal change in the rest of the ROIs was not significantly different in pre$\mathrm{AD}$ respect to controls.

We also examined other components from the ICA decomposition and found a secondary pattern that resulted significant in the post-hoc GLM analysis between groups. This component was associated with the task time series (i.e., activated during encoding blocks) and it was more activated in the pre-AD group ( $p<0.0033$ in the post-hoc regression analysis). The spatial map of the component included regions in the right insular and fronto-orbital cortices, the lingual gyrus, and the precuneus (Fig. 2)

\section{DISCUSSION}

In this study fMRI was used to investigate the functional brain activation pattern in pre-AD subjects during a visual encoding memory task. The main taskrelated component, found in all subjects (CTR and pre-AD), included the activation of visual associative areas and prefrontal executive regions and the deactivation of the default-mode network. An observed relevant finding was that pre-AD subjects presented a greater BOLD response scores compared to CTR within the precuneus and posterior cingulate cortex ROI during fixation, with a tendency for negative 


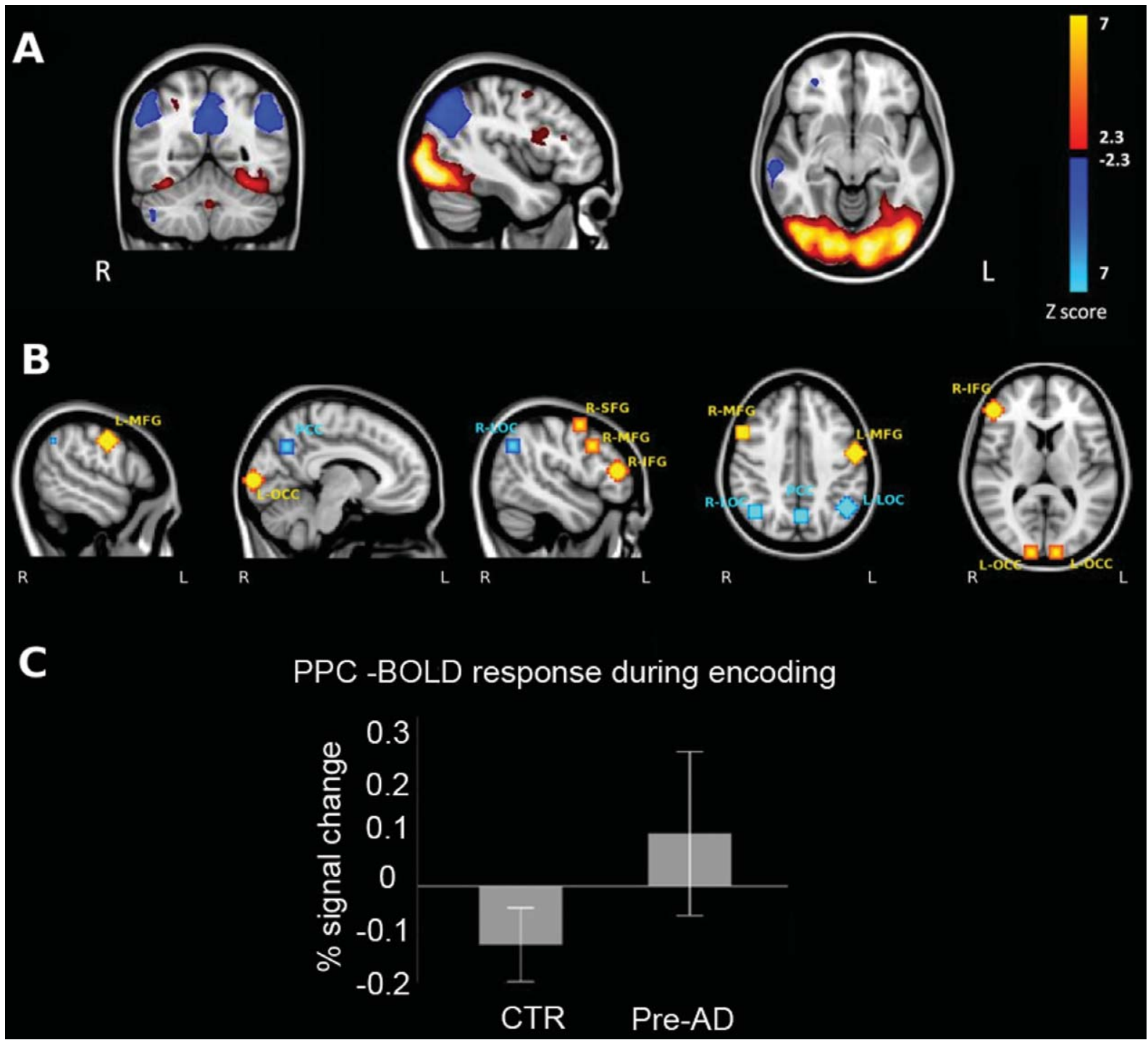

Fig. 1. A) Spatial map of the main independent component associated with the encoding condition. Positive (activated) areas are shown in red-yellow colors and negative (deactivated areas) in blue. B) Spherical ROIs created from the map above. C) Boxplot showing the mean activation (averaged BOLD response) in the precuneus and posterior cingulate cortex (PCC) for the two groups during encoding.

correlation between the activity in the precuneus and posterior cingulate cortex during encoding and the task performance.

The precuneus is a core region of the DMN [26] included in the posteromedial cortex, which has also been demonstrated to be a part of a memory system that is deactivated during successful encoding and activated during successful retrieval processes $[27,28]$. The changes we have observed in this region are prior to the appearance of observable memory decline in our pre-AD subjects. These results corroborate results described subtle functional changes during resting state in healthy subjects with positive PIB [12-15]. Recently, in a study of cognitively preserved subjects Sheline et al. [12] found that the $\mathrm{PIB}+$ group differed significantly from the PIB-group in terms of functional connectivity in the precuneus to hippocampus, parahippocampus, anterior cingulate, dorsal cingulate, gyrus rectus, superior precuneus, and visual cortex during resting state in fMRI. Also, Sperling et al. [13] demonstrated that high levels of amyloid deposition were associated with aberrant 
Table 2

Definition and localization of ROIs for fMRI analysis

\begin{tabular}{llcl}
\hline ROI id & Functional network & MNI coordinates & Brain area \\
\hline L-OCC & Task-positive & $(-10,-98,4)$ & Left occipital cortex \\
L-MFG & Task-positive & $(-54,-6,40)$ & Left middle frontal gyrus \\
R-OCC & Task-positive & $(14,-98,16)$ & Right occipital cortex \\
R-MFG & Task-positive & $(54,14,36)$ & Right middle frontal gyrus \\
R-IFG & Task-positive & $(50,38,12)$ & Right inferior frontal gyrus \\
R-SFG & Task-positive & $(42,2,56)$ & Right superior frontal gyrus \\
PPC & Task-negative & $(-2,-66,36)$ & Precuneus/posterior cingulate cortex \\
L-LOC & Task-negative & $(-46,-58,40)$ & Left lateral occipital cortex \\
R-LOC & Task-negative & $(42,-62,36)$ & Right lateral occipital cortex \\
\hline
\end{tabular}

L-OCC: left occipital cortex; L-MFG: left middle frontal gyrus; R-OCC: right occipital cortex; R-MFG: right middle frontal gyrus; R-IFG: right inferior frontal gyrus; R-SFG: right superior frontal gyrus; PPC: precuneus and posterior cingulate cortex; L-LOC: left lateral occipital cortex; R-LOC: right lateral occipital cortex.

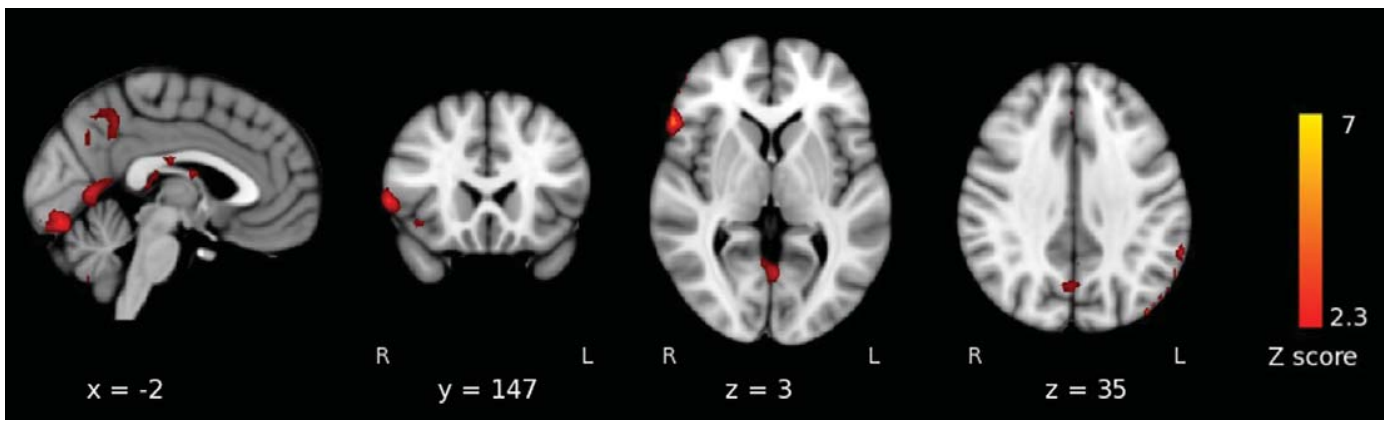

Fig. 2. Spatial map of the second component found from the independent component analysis decomposition.

default network fMRI activity, similar to the pattern of dysfunction reported in AD patients. Taking together, structural and functional changes in the precuneus and posterior cingulate cortex described in pre-AD subjects suggest that cerebral amyloidosis in pre-AD phases is associated with structural and functional changes in the medial parietal areas, core regions of the DMN $[8,26]$. In this line, recent findings using cross neuroimaging modality analyses in PIB positive healthy elders and MCI patients, confirmed that low metabolism in posterior cingulate cortex/precuneus regions related to reduced whole-brain connectivity measured with fMRI, suggesting that these cortical hub regions may be particularly vulnerable to neurodegeneration induced by elevated amyloid burden [16].

Taking together, current results and results from previous studies, may suggest that functional neocortical changes, mainly in the medial parietal regions occur in the preclinical phases of the disease while functional temporal dysfunction have consistently described in the clinical phases of AD [29, 30]. The fact that neocortical dysfunctions may be present before medial temporal lobe dysfunction in the AD continuum should be consolidated by future studies.

We observed hyperactivity in the precuneus and posterior cingulate cortex in the pre-AD group. In our sample, although there were no differences in the recognition task between CTR and pre-AD subjects, pre-AD subjects activated additional brain resources in order to perform at the same level as controls. Therefore, the greater BOLD response within precuneus and posterior cingulate cortex region found in the pre-AD subjects during fixation presented a tendency for negative correlation between the activity in the precuneus and posterior cingulate cortex during encoding and the task performance. Therefore, more hyperactivation is associated with high performance during the task. A hypothesis of hyperactivity regions during fMRI has been proposed by Sperling et al. [13], who speculated that the hyperactivity of some regions could be due to a decrease in synaptic inhibition, potentially modifying the functional properties of the neurons themselves, rendering them hyperactive. However, further data will be necessary in order to confirm if this neuron reaction it can be similar to the 
hyperactivity described in some regions like hippocampus, that might represent evidence of neuronal excitotoxicity, representing impending synaptic failure and incipient cognitive decline processing $[31,32]$.

As AD pathology begins decades before the manifestation of symptoms [8], diagnosis in the presymptomatic stage may become a reality. This has intensified the search for efficient preclinical AD biomarkers. The AD-modifying treatments developed in the future may well require very early diagnosis to optimize their potential benefits, and in order to show their efficacy future trials may have to target a population with a biological signature of the disease with minimal or even zero clinical symptomatology [33]. Although there is currently no consensus on the significance and prognostic value of AD pathological hallmarks detected in the preclinical state, recent studies from our group suggest that this phase may be clinically silent but "biologically" active, since the findings indicate structural MRI changes in both presymptomatic and pre-AD subjects without cognitive impairment $[6,7]$. We now extend this observation by showing distinct brain functional activity in pre$\mathrm{AD}$ subjects. How long CSF $\mathrm{A} \beta_{42}$ decrease needs to be present before brain circuitry change occurs is an important topic that should be investigated in longitudinal studies. Furthermore, longitudinal studies will be important to determine the time course of functional and structural brain changes in order to help plan the optimal timing for potential interventional studies in pre-AD individuals.

Some limitations should be considered in our study, mainly the fact that sample size is relatively small, due to the difficulties for including this type of subjects; however the main results survived a correction for multiple comparisons, making them more reliable. Also, we are aware that the methodology proposed in this study is exploratory and further studies with larger sample would be needed for obtaining confirmatory results about this group. Lack of longitudinal follow-up is other limitation of this work that has been acknowledged. In this sense, we want to emphasize that the time period to have sufficient power to detect a conversion to a dementia of the Alzheimer type (or even to MCI diagnosis) is very long. However, several studies have already shown that subjects with positive biomarker have more likely cognitive impairment and structural changes after several months of follow-up [34-37]. In this sense, although individually there is no assurance the development of dementia in these subjects, the risk of developing AD in this group is high. Therefore, we believe that cross-sectional studies as this one will continue to be necessary, in order to bring new hypothesis to the field.

In summary, our results show that pre-AD subjects presented increased activation in the precuneus and posterior cingulate cortex suggests that these are key regions of early brain dysfunction in the preclinical AD phases. These findings may represent that subtle changes in functional brain activity precede clinical and cognitive symptoms in the AD continuum. The potential value of fMRI as a biomarker of the preclinical AD state still needs to be demonstrated.

\section{ACKNOWLEDGMENTS}

This work was supported by Spanish Ministry of Science. Dr Lorena Rami is the recipient of a Miguel Servet grant as a senior investigator from the Spanish Ministry of Science (CP08/00147). This study was funded by research grants from the Spanish Ministerio de Ciencia e Innovación to Dr. David BartrésFaz (SAF2009-07489), to Dr. Lorena Rami (FIS PI11/01071), Fondo europeo de desarrollo regional, una manera de hacer Europa, grant from IMSERSO 197/2011, and a CONSOLIDER grant (CSD 201000045). Acknowledgments go to VALLVERD, S.A for their scientific economic support. All authors report no biomedical financial interests or potential conflicts of interest. We thank all the volunteers for participating in this study.

Authors' disclosures available online (http://www.jalz.com/disclosures/view.php?id=1288).

\section{REFERENCES}

[1] Shaw LM, Vanderstichele H, Knapik-Czajka M, Clark CM, Aisen PS, Petersen RC, Blennow K, Soares H, Simon A, Lewczuk P, Dean R, Siemers E, Potter W, Lee VM, Trojanowski JQ (2009) Cerebrospinal fluid biomarker signature in Alzheimer's disease neuroimaging initiative subjects. Ann Neurol 65, 403-413.

[2] Jack CR, Knopman DA, Jagust WJ, Shaw LM, Aisen PS, Weiner MW, Petersen RC, Trojanowski JQ (2010) Hypothetical model of dynamic biomarkers of the Alzheimer's pathological cascade. Lancet Neurol 9, 119-128.

[3] Iacono D, O'Brien R, Resnick SM, Zonderman AB, Pletnikova O, Rudow G, An Y, West MJ, Crain B, Troncoso JC (2008) Neuronal hypertrophy in asymptomatic Alzheimer disease. J Neuropathol Exp Neurol 67, 578-589.

[4] Mintun MA, Larossa GN, Sheline YL, Dence CS, Lee SY, Mach RH, Klunk WE, Mathis CA, DeKosky ST, Morris JC (2006) [11C]PIB in nondemented population: Potential antecedent marker of Alzheimer disease. Neurology 67, 446452.

[5] Price J, Morris J (1999) Tangles and plaques in nondemented aging and "preclinical" Alzheimer's disease. Ann Neurol 45, 358-368. 
[6] Fortea J, Sala-Llonch R, Bartrés-Faz D, Bosch B, Lladó A, Bargalló N, Molinuevo JL, Sánchez-Valle R (2010) Increased cortical thickness and caudate volume precede atrophy in PSEN1 mutation carriers. J Alzheimers Dis 22, 909-922.

[7] Fortea J, Sala-Llonch R, Bartrés-Faz D, Lladó A, SoléPadullés C, Bosch B, Antonell A, Olives J, Sanchez-Valle R, Molinuevo JL, Rami L (2011) Cognitively preserved subjects with transitional CSF $\beta$-amyloid 1-42 values have thicker cortex in AD vulnerable areas. Biol Psychiatry 70, 183-190.

[8] Sperling RA, Aisen PS, Beckett LA, Bennett DA, Craft S, Fagan AM, Iwatsubo T, Jack CR Jr, Kaye J, Montine TJ, Park DC, Reiman EM, Rowe CC, Siemers E, Stern Y, Yaffe K, Carrillo MC, Thies B, Morrison-Bogora M, Wagster MV, Phelps $\mathrm{CH}$ (2011) Toward defining the preclinical stages of Alzheimer's disease: Recommendation from the national institute of aging-Alzheimer's association workgroups on diagnostic guidelines for Alzheimer's disease. Alzheimers Dement 7, 280-292.

[9] Dubois B, Feldman HH, Jacova C, Cummings JL, Dekosky ST, Barberger-Gateau P, Delacourte A, Frisoni G, Fox NC, Galasko D, Gauthier S, Hampel H, Jicha GA, Meguro K, O'Brien J, Pasquier F, Robert P, Rossor M, Salloway S, Sarazin M, de Souza LC, Stern Y, Visser PJ, Scheltens P (2010) Revising the definition of Alzheimer's disease: A new lexicon. Lancet Neurol 9, 1118-1127.

[10] Rami L, Fortea J, Bosch B, Solé-Padullés C, Lladó A, Iranzo A, Sánchez-Valle R, Molinuevo JL (2011) Cerebrospinal fluid biomarkers and memory present distinct associations along the continuum from healthy subjects to AD patients. $J$ Alzheimers Dis 23, 319-326.

[11] Prvulovic D, Bokde ALW, Faltraco F, Hampel H (2011) Functional magnetic resonance imaging as a dynamic candidate biomarker for Alzheimer's disease. Prog Neurobiol 95, 557 569.

[12] Sheline YI, Raichle ME, Snyder AZ, Morris JC, Head D, Wang S, Mintun MA (2010) Amyloid plaques disrupt resting state default mode network connectivity in cognitively normal elderly. Biol Psychiatry 67, 584-587.

[13] Sperling RA, Laviolette PS, O'Keefe K, O'Brien J, Rentz DM, Pihlajamaki M, Marshall G, Hyman BT, Selkoe DJ, Hedden T, Buckner RL, Becker JA, Johnson KA (2009) Amyloid deposition is associated with impaired default network function in older persons without dementia. Neuron 63, 178-188.

[14] Hedden T, Van Dijk KR, Becker JA, Mehta A, Sperling RA, Johnson KA, Buckner RL (2009) Disruption of functional connectivity in clinically normal older adults harboring amyloid burden. J Neurosci 29, 12686-12694.

[15] Nelissen N, Vandenbulcke M, Fannes K, Verbruggen A, Peeters R, Dupont P, Van Laere K, Bormans G, Vandenberghe $\mathrm{R}$ (2007) Abeta amyloid deposition in the language system and how the brain responds. Brain 130, 2055-2069.

[16] Drzezga A, Becker JA, Van Dijk KRA, Screenivasa A, Talukdar T, Sullivan C, Schultz AP, Sepulcre J, Putcha D, Greve D, Johnson KA, Sperling RA (2011) Neuronal dysfunction and disconnection of cortical hubs in non-demented subjects with elevated amyloid burden. Brain 134, 1635-1646.

[17] Fagan AM, Head D, Shah AR, Marcus D, Mintun M, Morris JC, Holtzman DM (2009) Decreased cerebrospinal fluid Abeta(42) correlates with brain atrophy in cognitively normal elderly. Ann Neurol 65, 176-183.

[18] Antonell A, Fortea J, Rami L, Bosch B, Balasa M, SánchezValle R, Iranzo A, Molinuevo J1, Lladó A (2011) Different profiles of Alzheimer's disease cerebrospinal fluid biomarkers in controls and subjects with subjective memory complaints. J Neural Transm 118, 259-262.
[19] Rami L, Gómez-Ansón B, Bosch B, Sánchez-Valle R, Monte GC, Villar A, Molinuevo JL (2007) Cortical brain metabolism as measured by proton spectroscopy is related to memory performance in patients with amnestic mild cognitive impairment and Alzheimer's disease. Dement Geriatr Cogn Disord 24, 274-279.

[20] Solé-Padullés C, Bartrés-Faz D, Junqué C, Vendrell P, Rami L, Clemente IC, Bosch B, Villar A, Bargalló N, Jurado MA, Barrios M, Molinuevo JL (2009) Brain structure and function related to cognitive reserve variables in normal aging, mild cognitive impairment and Alzheimer's disease. Neurobiol Aging 30, 1114-1124.

[21] Beckmann CF, Smith SM (2005) Tensorial extensions of independent component analysis for multisubject FMRI analysis. Neuroimage 25, 294-311.

[22] Jenkinson M, Bannister P, Brady M, Smith S (2002) Improved optimization for the robust and accurate linear registration and motion correction of brain images. Neuroimage 17, 825-841.

[23] Smith SM (2002) Fast robust automated brain extraction. Hum Brain Mapp 17, 143-155.

[24] Woolrich MW, Ripley BD, Brady JM, Smith SM (2001) Temporal autocorrelation in univariate linear modelling of FMRI data. NeuroImage 14, 1370-1386.

[25] Buckner RL, Andrews-Hanna JR, Schacter DL (2008) The brain's default network: Anatomy, function, and relevance to disease. Ann N Y Acad Sci 1124, 1-38.

[26] Raichle ME, MacLeod AM, Snyder AZ, Powers WJ, Gusnard DA, Shulman GL (2001) A default mode of brain function. Proc Natl Acad Sci U S A 16, 676-682.

[27] Daselaar SM, Prince SE, Cabeza R (2004) When less means more: Deactivations during encoding that predict subsequent memory. Neuroimage 23, 921-927.

[28] Vannini P, O’Brien J, O'Keefe K, Pihlajamäki M, Laviolette P, Sperling RA (2011) What goes down must come up: Role of the posteromedial cortices in encoding and retrieval. Cereb Cortex 21, 22-34.

[29] Celone KA, Calhoun VD, Dickerson BC, Atri A, Chua EF, Miller SL, DePeau K, Rentz DM, Selkoe DJ, Blacker D, Albert MS, Sperling RA (2006) Alterations in memory networks in mild cognitive impairment and Alzheimer's disease: An independent component analysis. J Neurosci 26, 1022210231.

[30] Sperling RA, Bates JF, Chua EF, Cocchiarella AJ, Rentz DM, Rosen BR, Schacter DL, Albert MS (2003) fMRI studies of associative encoding in young and elderly controls and mild Alzheimer's disease. J Neurol Neurosurg Psychiatry 74, 4450 .

[31] Miller SL, Fenstermacher E, Bates J, Blacker D, Sperling RA, Dickerson BC (2008) Hippocampal activation in adults with mild cognitive impairment predicts subsequent cognitive decline. J Neurol Neurosurg Psychiatry 79, 630-635.

[32] O'Brien JL, O'Keefe KM, LaViolette PS, DeLuca AN, Blacker D, Dickerson BC, Sperling RA (2010) Longitudinal fMRI in elderly reveals loss of hippocampal activation with clinical decline. Neurology 74, 1969-1976.

[33] Winblad B, Wimo A, Engedal K, Soininen H, Verhey F, Waldemar G, Wetterholm AL, Haglund A, Zhang R, Schindler $\mathrm{R}$ (2006) 3-year study of donepezil therapy in Alzheimer's disease: Effects of early and continuous therapy. Dement Geriatr Cogn Disord 21, 353-363.

[34] Schott JM, Bartlett JW, Fox NC, Barnes J (2010) Alzheimer's Disease Neuroimaging Initiative Investigators. Increased brain atrophy rates in cognitively normal older adults with low cerebrospinal fluid A $\beta 1-42$. Ann Neurol 68 , 825-834. 
[35] Vemuri P, Wiste HJ, Weigand SD, Shaw LM, Trojanowski JQ, Weiner MW, Knopman DS, Petersen RC, Jack CR Jr, Alzheimer's Disease Neuroimaging, Initiative (2009) MRI and CSF biomarkers in normal, MCI, and AD subjects: Predicting future clinical change. Neurology 73, 294-301.

[36] Tosun D, Schuff N, Truran-Sacrey D, Shaw LM, Trojanowski JQ, Aisen P, Peterson R, Weiner MW, Alzheimer's Disease
Neuroimaging, Initiative (2010) Relations between brain tissue loss, CSF biomarkers, and the ApoE genetic profile: A longitudinal MRI study. Neurobiol Aging 31, 1340-1354.

[37] Fagan AM, Roe CM, Xiong C, Mintun MA, Morris JC, Holtzman DM (2007) Cerebrospinal fluid tau/beta-amyloid(42) ratio as a prediction of cognitive decline in nondemented older adults. Arch Neurol 64, 343-349. 